\title{
4 Zur Unterscheidung zwischen west- und ostslawischen Aspektsystemen
}

\subsection{Einführung}

Dickey hat in seiner Studie Parameters of Slavic Aspect (2000) gezeigt, dass es einen intratypologischen Unterschied zwischen den west- und ostslawischen Sprachen gibt, der mit dem unterschiedlichen Grad der Aspektgrammatikalisierung zusammenhängt. Nach Dickey zählen Tschechisch, Slowenisch, Slowakisch und Sorbisch zu der westslawischen Gruppe, während Russisch, Ukrainisch, Weißrussisch und Bulgarisch in die ostslawische Gruppe gehören. Tschechisch bzw. Russisch gelten dabei als Prototypen der jeweiligen Gruppe. Polnisch und Serbokroatisch stellen eine Übergangszone zwischen diesen beiden Aspektsystemen dar.

Die Grundidee dieser Unterscheidung ist, dass sich innerhalb der slawischen Sprachen eine diachrone Entwicklung vollzog, die dazu führte, dass in den ostslawischen Sprachen die Aspektkategorie stärker grammatikalisiert wurde. Dies ging mit einer bestimmten Regularisierung des Aspektgebrauchs in Abhängigkeit von einem bestimmten Situationstyp einher. Beispielsweise ist im Russischen für iterative Handlungen die Verwendung des imperfektiven Aspekts nahezu obligatorisch. In den westslawischen Systemen dagegen erfolgte eine solche Regularisierung der Verwendungsbedingungen in einem viel kleineren Umfang, so dass in vielen Kontexten die Verwendung beider Aspekte möglich ist (z.B. für wiederholte Handlungen oder Handlungssequenzen sowie beim Gebrauch vom des historischen Präsens). In dem westslawischen Sprachentyp können darüber hinaus auch Verbalnomina aspektuell markiert werden. ${ }^{78}$

\subsection{Die Studie}

Wie bereits erwähnt, wurden die Unterschiede zwischen dem Tschechischen und Russischen, die in den früheren Studien (z.B. Kap. 5) festgestellt wurden, auch in Verbindung mit der These von Dickey (2000) interpretiert. Es stellt sich jedoch die Frage, ob auch andere Sprachen, die nach Dickey der westlichen Sprachgruppe

78 Zum Beispiel: tsch. hození míče na trávu vs. vyhození/vyhazování míče do vzduchu 'das Werfen eines Balles aufs Gras' vs. 'das Raus- oder Hochwerfen eines Balles' in die Luft einmalig/ mehrmalig (vgl. Schmiedtová 2013a; Kap. 3). 
angehören, in dem hier verwendeten experimentellen Design in die gleiche Gruppe wie das Tschechische fallen würden. Um dies $\mathrm{zu}$ überprüfen, wurden neue Daten von polnischen ${ }^{79}(\mathrm{~N}=30)$ und slowakischen ${ }^{80}(\mathrm{~N}=15)$ Muttersprachlern herangezogen und mit den bereits vorhandenen Daten aus dem Tschechischen, Russischen und Deutschen ${ }^{81}$ verglichen.

Der Gegenstand der Untersuchung war die Enkodierung von zielorientierten Bewegungsereignissen, bei der insbesondere die Kodierung der Endpunkte im Fokus war. Es wurden drei Arten von Stimuli verwendet: 1) Kritische Stimuli ( $n=12$ ) waren zielorientierte Bewegungsereignisse, in denen eine Bewegung belebter Entitäten (Menschen oder Tiere) von Punkt A zu Punkt B als nicht erreicht gezeigt wurde. 2) Kontrollstimuli waren ebenfalls zielorientierte Bewegungsereignisse, in denen die Bewegung als erreicht dargestellt wurde $(n=12)$. Für die kritischen Stimuli wurden zwischen den getesteten Sprachen Unterschiede erwartet, für die Kontrollstimuli nicht. 3) Außerdem wurden sogenannte Filler $(n=36)$ gebraucht, die homogene Aktivitäten oder statische Situationen darstellten. Die Filler dienten der Ablenkung.

\subsection{Ergebnisse}

Abbildung 11 fasst die Ergebnisse der Analysen der Endpunktmarkierung in den kritischen und Kontrollszenen für alle fünf Sprachen zusammen. Ihr ist zu entnehmen, dass tschechische Sprecher bedeutend mehr Endpunkte für die Darstellung der kritischen Szenen [-END] erwähnen, als dies russische, polnische und slowakische Sprecher tun. Tschechische Sprecher gehen also so vor, dass sie Endpunkte in allen Szenen (da, wo ein Endpunkt inferiert werden muss) in der Mehrheit der Äußerungen verbalisieren. Auch deutsche Sprecher erwähnen in allen kritischen Szenen [-END] mehr Endpunkte als russische, polnische und slowakische Sprecher. Die Präferenz für die Endpunktmarkierung der deutschen und tschechischen Sprecher ist vergleichbar und liegt im Durchschnitt bei 63\%.

79 Die polnischen Daten wurden im Rahmen der Magisterarbeit von Fedus (2006) erhoben. Die hier präsentierten Ergebnisse basieren auf einer kürzlich durchgeführten Neuanalyse dieser Daten. Vielen Dank an Sylwia Obrebska für die Hilfe bei der Datenanalyse.

80 Ich danke ganz herzlich Norbert Vaněk für die Erhebung der slowakischen Daten.

81 Die deutschen, tschechischen und russischen Daten stammen aus einer früheren Studie (siehe Kap. 5). 


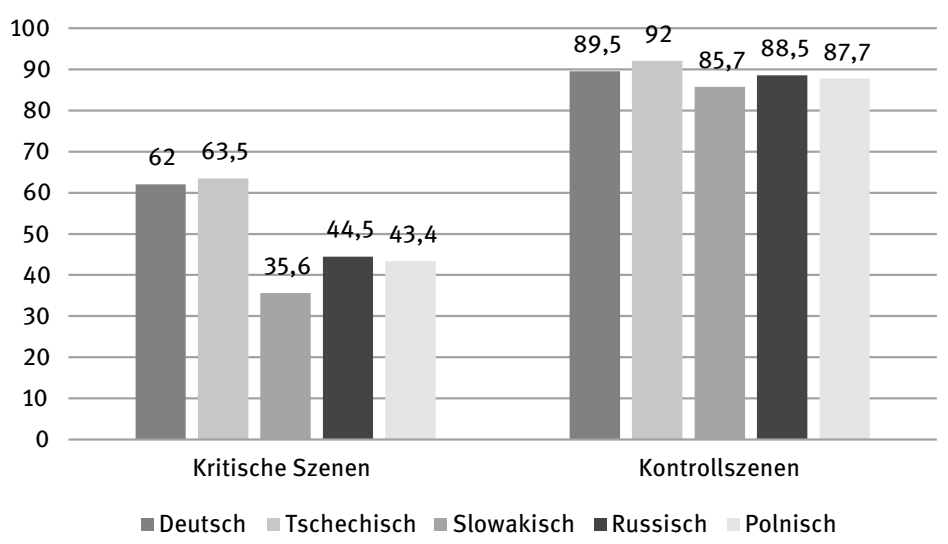

Abb. 11: Enkodierung von Endpunkten im Deutschen, Tschechischen, Slowakischen, Russischen und Polnischen

Sprecher des Russischen, Polnischen und Slowakischen scheinen dagegen eine andere Vorgehensweise zu haben: Sie erwähnen bevorzugt erst dann die Endpunkte, wenn diese in einem Clip auch tatsächlich als erreicht gezeigt werden [+END], d.h. vorwiegend in den Kontrollszenen. In den kritischen Szenen [-END] dagegen erwähnen Sprecher dieser drei Sprachen deutlich weniger Endpunkte (im Durchschnitt 41\%) und unterscheiden sich dadurch von Sprechern des Tschechischen und Deutschen. ${ }^{82}$

Für Kontrollszenen, in denen der Endpunkt als erreicht dargestellt wird [+END], wurden für die vorliegenden Daten keine statistisch relevanten Unterschiede festgestellt.

Diese Ergebnisse zeigen, dass polnische Sprecher bei der Enkodierung von zielorientieren Bewegungsereignissen nicht den potenziellen Endpunkt, sondern viel mehr (in etwa 73\% aller relevanten Äußerungen) den Bewegungsverlauf (Weg) fokussieren (vgl. für längere narrative Texte Śledzka 2007). In den polnischen Daten sind viele Beschreibungen mit Verlaufsinformation vorzufinden: z.B. pol. Pociag jedzie po torach ('Der Zug fährt auf Gleisen'); zum Teil auch in Nominalform pol. jadacy pociagg ('ein fahrender Zug/der fahrende Zug'). So fällt das Polnische in die gleiche Gruppe wie das Russische, das ebenfalls für ziel-

82 Zum Vergleich deutscher und tschechischer Tempus-Aspekt-Systeme vgl. Schmiedtová (2003b, 2004); Kapitel 1 und 8. 
orientierte Bewegungsereignisse die Verlaufsperspektive bevorzugt verwendet (siehe z.B. Kap. 8).

Auch in den slowakischen Daten wird in den kritischen Szenen der Verlauf der dargestellten Bewegung enkodiert. Neben der Enkodierung des Wegs, welche in 35\% aller Äußerungen vorkommt, werden in den kritischen Szenen auch Endpunkte $(35,6 \%)$ sowie in einem etwas geringen Maße (23,1\%) Information zum Ort der Bewegung zum Ausdruck gebracht. Diese Befunde könnten mit der relativ kleinen Größe des Datensets zusammenhängen. Es wäre aber zu erwarten, dass bei einer größeren Probandenzahl die Wahrscheinlichkeit besteht, dass sich eine bestimmte Präferenz herausbildet. In Anbetracht der Unterschiede in der Standardabweichung $(\mathrm{StAb})^{83}$ für die Enkodierung der Endpunkte $(\mathrm{StAb}=2.6)$ und des Weges $(\mathrm{StAb}=1.4)$ ist anzunehmen, dass sich im Slowakischen eine Präferenz für Trajektoriemarkierung (path) entwickeln könnte.

Des Weiteren ist für diese Arbeit die Gegenüberstellung der slowakischen und der tschechischen Daten relevant. Diese findet sich in Abbildung 12.

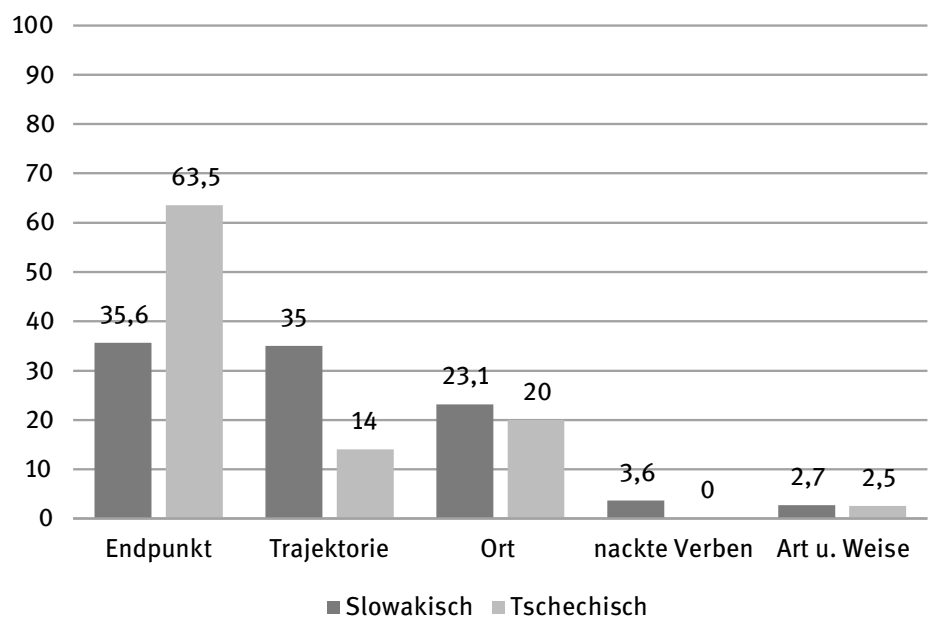

Abb. 12: Enkodierung zielorientierter Bewegungsereignisse (kritische Szenen) durch slowakische und tschechische Muttersprachler

83 Die Standardabweichung (StAb) weist auf die Varianz in den Daten hin. Das heißt, je kleiner die StAb, desto weniger Varianz in den Daten. Im Hinblick auf die slowakischen Daten bedeutet dies, dass in der Enkodierung der Trajektorie (path) die Probanden ein viel homogeneres Verhalten zeigen als in der Enkodierung der Endpunkte. 
Abbildung 12 zeigt, dass tschechische Sprecher in den kritischen Szenen überwiegend häufig den Endpunkt enkodieren und somit die Ereignisse unter der sogenannten holistischen Perspektive darstellen (vgl. Schmiedtová/von Stutterheim/Carroll 2011; Kap. 5). Im Tschechischen wird der Endpunkt signifikant häufiger als im Slowakischen erwähnt $(\mathrm{z}=1,98, p<.05)$. Ein entgegengesetzter Trend zeigt sich im Bereich des Wegs: Die Enkodierung des Wegs kommt in den slowakischen Daten 2,5-mal öfter vor als in den tschechischen Daten. Auch dieser Unterschied ist statistisch relevant $(\mathrm{z}=2,04, p<.05)$.

Erwähnenswert ist außerdem der Unterschied im Gebrauch der sogenannten nackten Verben (vgl. Schmiedtová 2011a, 2013a). Hier handelt es sich um Konstruktionen, die nur aus Subjekt und Verb bestehen, z.B. Ein Auto fährt oder Zwei Frauen laufen. Der prozentuale Anteil im Slowakischen liegt zwar nur bei 3,6\%, in den tschechischen Daten kommen nackte Verben in diesen Daten aber überhaupt nicht vor. ${ }^{84}$ Im Hinblick auf den Ausdruck des Ortes (z.B. auf der Straße gehen, in der Altstadt laufen) sowie der Art und Weise (z.B. schnell fahren, gemütlich gehen) lassen sich für den tschechisch-slowakischen Vergleich keine Unterschiede feststellen.

\subsection{Fazit und Diskussion}

In Anbetracht dieser Ergebnisse und der Befunde früherer Studien zum Zusammenhang von Endpunktkodierung und Aspektmarkierung (vgl. Carroll/von Stutterheim/Nüse 2004; Kap. 5-9) lässt sich die Vermutung aufstellen, dass die von Dickey postulierte Trennungslinie zwischen ost- und westslawischen Sprachen möglicherweise weiter verfeinert werden muss, indem sie als eine Art Skala interpretiert und zugleich die Auswirkung eines möglichen Sprachkontakts in Rechnung gestellt wird.

Dies machen insbesondere die Analysen der slowakischen Daten deutlich, welche zeigen, dass das Slowakische bei der Enkodierung von Bewegungsereignissen nicht wie das Tschechische auf die holistische Perspektive unter Einbezug der Endpunkte greift, sondern auch andere Informationen, vor allem den Weg, zum Ausdruck bringt. Es ist bemerkenswert, dass diese Tendenzen bereits anhand einer relativ kleinen Stichprobe identifiziert werden konnten. Nichtsdestotrotz bedarf es weiterer empirisch gestützter sowie theoretisch fundierter

84 In den russischen Daten liegt der Gebrauch der nackten Verben bei 13\% und steigt auf 20\%, wenn Daten unter Zeitdruck elizitiert werden (Schmiedtová 2013a). Nackte Verbalphrasen finden sich vermehrt auch in L2-Daten von russischen Sprechern des Deutschen (Schmiedtová 2011a). 
vergleichender Forschungsarbeiten zum Slowakischen, um genau festzustellen, welche Perspektivierungsmuster im Slowakischen gebräuchlich sind (vgl. Januška 2017 zum Einfluss des Ungarischen auf das Slowakische).

Darüber hinaus wäre es wünschenswert, experimentelle Daten auch aus dem Slowenischen und Sorbischen heranziehen zu können, um die möglichen aspektuellen Unterschiede und die Konsequenzen dieser Unterschiede für die Perspektivierung bei Ereignisenkodierung innerhalb der westslawischen Gruppe näher untersuchen zu können.

Die Befunde zum Slowakischen und zum Polnischen sind auch für die Diskussion über die Reichweite des deutschen Spracheinflusses relevant. Zweifellos standen auch diese beiden Sprachen im Kontakt zum Deutschen. So könnte man auf den ersten Blick vermuten, dass sich im Polnischen und Slowakischen das deutsche System auf eine ähnliche Art und Weise auswirkte, wie es im Tschechischen der Fall war. Die Datenlage zeigt mit zunehmender Deutlichkeit, dass die geografische Nähe zweier Sprachen alleine nicht hinreichend ist, um Änderungen in einem Sprachsystem auszulösen (vgl. Matras 2009). Das Deutsche hatte beispielsweise auf dem böhmischen Gebiet einen anderen Stellenwert als im polnischen oder slowakischen Raum (vgl. Newerkla 2011). Dies könnte möglicherweise dazu geführt haben, dass sich das Deutsche in einem anderen Ausmaß auf das Tschechische als auf das Polnische und Slowakische ${ }^{85}$ ausgewirkt hat.

Was macht also den deutsch-tschechischen Kontakt so besonders? Im Rahmen der vorliegenden Arbeit, die den Einfluss synchron und anhand von empirischen Daten untersucht, lässt sich diese Frage nicht umfassend beantworten. Mit Sicherheit kann man jedoch festhalten, dass der über Jahrhunderte andauernde deutsch-tschechische Kontakt in vielen tschechischen Gebieten zu funktionaler Bilingualität führte (vgl. Trost 1965; Fürst 2003), die es in dem Ausmaß in den anderen zwei Ländern nicht gegeben hat. Die gelebte Zweisprachigkeit, der enge kulturelle Kontakt und Austausch zwischen den beiden Sprachen sowie viele andere wichtige psychosoziale und soziolinguistische Faktoren waren maßgeblich für die Formung und Entwicklung des tschechischen Sprachsystems, deren Auswirkungen auch im heutigen Tschechisch zu beobachten sind.

Diese Arbeit stellt eine der ersten systematischen und empirisch gestützten Forschungen zu diesem Thema dar, welches aber unbedingt - insbesondere in Bezug auf die Schnittstelle zwischen Sprachkontakt und Zeitgeschichte unter Berücksichtigung der Psycholinguistik - weiter untersucht werden müsste.

85 Für die Untersuchung des deutsch-slowakischen Kontakts sind sicherlich zwei Faktoren von Belang: (1) Die eher mangelnde Schriftlichkeit auf dem slowakischen Gebiet zur Zeit des Sprachkontakts; (2) der Spracheinfluss aus dem Ungarischen. 


\subsection{Zusammenfassung}

In Kapitel 4 werden Elizitationsdaten aus weiteren westslawischen Sprachen präsentiert und in Bezug auf die Hypothese zur Unterscheidung der west- und ostslawischen Aspektsysteme (Dickey 2000) diskutiert. Die Ergebnisse zeigen, dass das Slowakische und Polnische bei der Enkodierung von Ereignissen anderen Konzeptualisierungsschemata als das Tschechische folgen. Vor allem die Daten aus dem Slowakischen deuten darauf hin, dass die Abgrenzung der west- von den ostslawischen Aspektsystemen im Sinne einer Skala zu betrachten ist und dass die Auswirkungen eines möglichen Sprachkontakts in typologischen Diskussionen (synchron und diachron) mitberücksichtigt werden müssen. 
